\title{
Actinomyces abscess mimicking mandibular bone cyst
}

\begin{abstract}
A radiolucent, well demarcated right mandibular bone lesion with a clinical presumptive diagnosis of solitary bone cyst was surgically excised in a 60year old woman. The histopathologic examination revealed Actinomyces colonies surrounded by chronic granulation tissue. This presentation is unique due to its completely encapsulated abscess formation rather than the more common fistula formation seen in Actinomyces lesions.
\end{abstract}

Keywords: actinomyces, histopathology, mandibular, abscess
Volume 3 Issue 5 - 2018

\author{
Nasuhi Engin Aydin \\ Department of Pathology, Ataturk Hospital, Turkey
}

Correspondence: Naushi Engin Aydin, Department of Pathology, Ataturk Hosiptal Karabaglar, Izmir, Turkey- 35000,Tel +90(546)6I57058, Email nasuhiengin@gmail.com

Received: December 20, 2018 | Published: December 28, 2018

\section{Introduction}

Actinomycosis is an infection with the anaerobic gram-positive filamentous bacterium Actinomyces, which may cause cervicofacial infection, and occasionally pulmonary, gastrointestinal, or disseminated infections. ${ }^{1,2}$ It is uncommon, but is still an important cause of morbidity. Its clinical presentation is usually indolent and chronic as slow growing masses that may evolve into fistulae, and for that reason are frequently underdiagnosed. Actinomyces spp is often disregarded clinically and is classified as a colonizing microorganisms since they are commensal in human body.

\section{Case presentation}

A 60 year old woman complaining of intermittent pain in the right jaw region for a few months was seen at the outpatient department. The patient's medical history was unremarkable without any health problem. Physical examination and $\mathrm{CBC}$ was unremarkable but a plain radiograph of the jaw region showed a $2 \mathrm{~cm}$ well delineated lucency suggestive of a simple bone cyst. A diagnostic and therapeutic surgical excision was made by curettage of the lesion. There was no cyst fluid, but soft friable tissue fragments with yellowish foci were noticed in the mandibular bone during the procedure. Specimens received at the pathology laboratory measured soft, light brown tissue fragments $1.5 \times 1 \times 1 \mathrm{~cm}$ in aggregate with yellowish areas. Histopathologic examination after paraffin embedding all the tissues received revealed a chronic inflammatory granulation tissue and huge, prominent colonies of a filamentous microorganism within small bone fragments (Figure 1) (Figure 2). The filamentous microorganism granules were Gram's stain and Grocott's methenamine silver (GMS) positive (Figure 3) besides yielding a periodic acid Schiff positive reaction following diastase digestion (Figure 4). A diagnosis of Actinomyces abscess was made. The aerobic microbiological cultures of the lesion was negative for any bacteria.

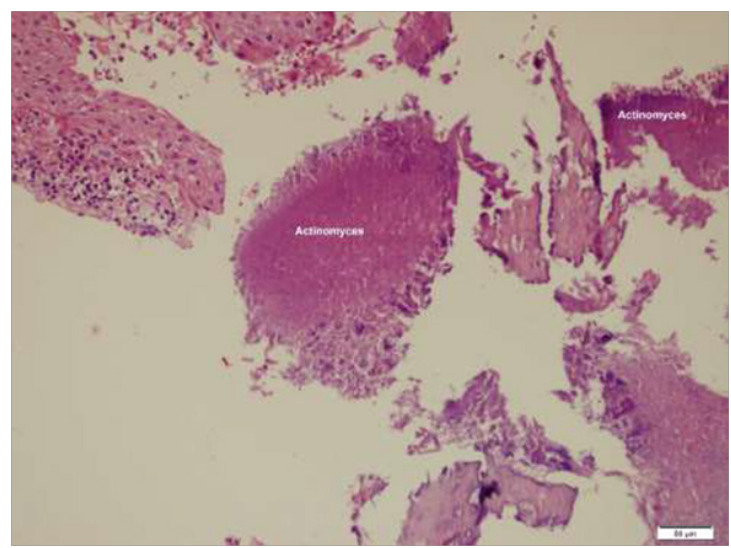

Figure I Huge groups of microrganisms among necrotic bone spicules and chronic inflammatory tissue elements, (Hematoxylin eosinx200).

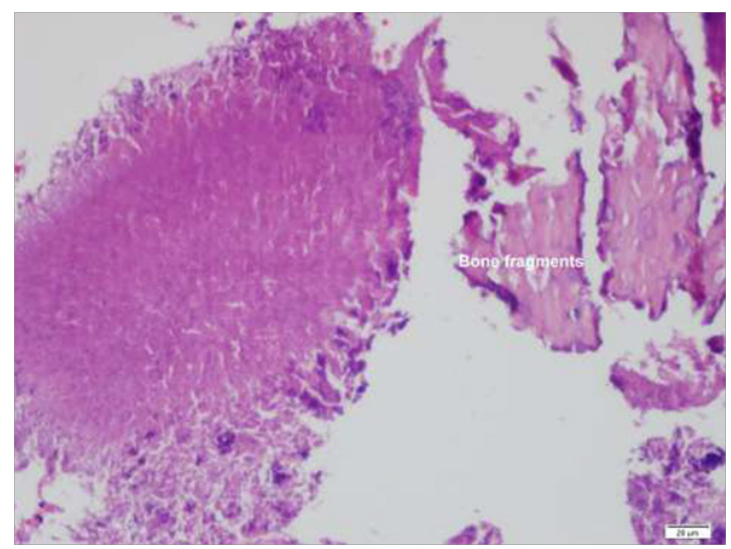

Figure 2 Higher power view of the microorganism croup showing a dense structure, ((Hematoxylin eosin $\times 400)$ 


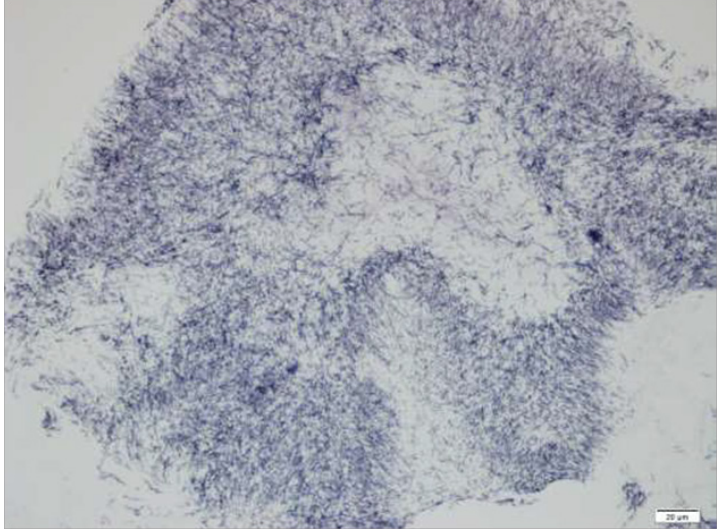

Figure 3 Silver impregnation of the sections clearly demonstrate the filamentous structure of the microorganism colony (GMSx400).

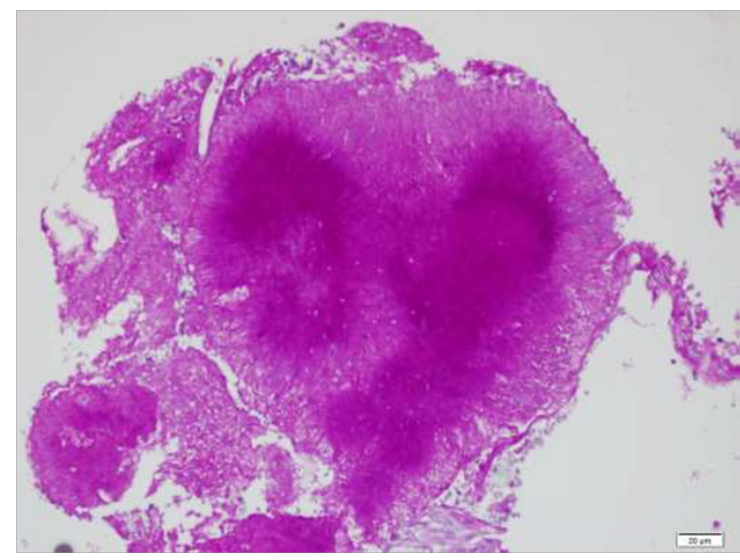

Figure 4 Magenta colored positive periodic acid Schiff reaction of the colony following diastase, (D-PASx400).

\section{Discussion/comment}

Due to its prominent filamentous morphology, this anaerobe microorganism was previously classified as a fungus but presently it is considered as a bacterium of the genus Actinomyces is a part of the oral, gastrointestinal and urogenital commensal flora belong to the Actinobacteria phylum and Actinomycetales order and are related to other genera such as Corynebacterium, Mycobacterium, Nocardia, and Propionibacterium. ${ }^{1,2}$ Physicians must be aware of typical clinical presentations (such as cervicofacial actinomycosis following dental focus of infection, pelvic actinomycosis in women with an intrauterine device, and pulmonary actinomycosis in smokers with poor dental hygiene), but also that actinomycosis may mimic neoplastic processes in various anatomical sites. ${ }^{1-3}$ In the human oropharynx, Actinomyces species are particularly prevalent within gingival crevices, tonsillar crypts, periodontal pockets and dental plaques, as well as on carious teeth. Consequently, actinomycosis is mainly considered an endogenous infection that is triggered by a mucosal lesion. ${ }^{4,5}$ However, the pathogenesis of invasive disease following oral mucosal breach is not clear. Cervicofacial actinomycosis is the most frequent clinical form that may be associated with large abscesses and/or mandibular osteomyelitis with or without sinus tract. Cervicofacial actinomycosis may lead to distant organ dissemination, including brain, lungs, and digestive tract so early diagnosis and treatment with penicillin group of antibiotics essential in which high-dose penicillin is the cornerstone of therapy. The risk of developing penicillin resistance appears to be minimal.

\section{Acknowledgments}

None.

\section{Conflicts of interest}

The authors declare that they have no conflicts of interest.

\section{References}

1. Mahadevia P, Brandwein-Gensler M. Infectious diseases of the head and neck in Surgical Pathology of Head and Neck. volume 3, Editor: Leon Barnes, 3rd ed. Informa Healthcare. 2009. p.1620-1621.

2. Valour F, Sénéchal A, Dupieux C, et al. Actinomycosis: etiology, clinical features, diagnosis, treatment, and management. Infection and Drug Resistance. 2014;7:183-197.

3. Isik B, Aydin E, Sogutlu G, et al. Abdominal actinomycosis simulating malignancy of the right colon. Dig Dis Sci. 2005;50(7):1312-1314.

4. Valour F, Chidiac C, Ferry T. A 22-year-old woman with right lumpy jaw syndrome and fistula. BMJ Case Rep Nov. 2015.

5. Gannepalli A, Ayinampudi BK, Baghirath PV, et al. Actinomycotic osteomyelitis of maxilla presenting as oroantral fistula. Case Rep Dent. 2015: 689240 